M. M. Mohie EL-Din - M. M. Amein • Nahed S. A. Ali •

M. S. Mohamed

\title{
Measures of information for concomitants of generalized order statistics from subfamilies of Farlie-Gumbel- Morgenstern distributions
}

Received: 6 November 2014 / Accepted: 6 August 2015 / Published online: 26 August 2015

(C) The Author(s) 2015. This article is published with open access at Springerlink.com

\begin{abstract}
In this paper, we study Shannon's entropy and Fisher information number for concomitants of generalized order statistics from subfamilies of Farlie-Gumbel-Morgenstern when the marginal distributions are Weibull, exponential, Pareto and power function. Also, we provide some numerical results of Shannon entropy and Fisher information number for concomitants of order statistics.
\end{abstract}

Mathematics Subject Classification $\quad 62 \mathrm{~B} 10 \cdot 62 \mathrm{H} 99 \cdot 62 \mathrm{P} 99$

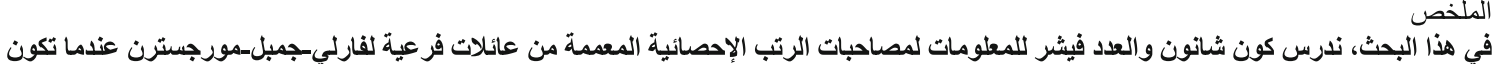

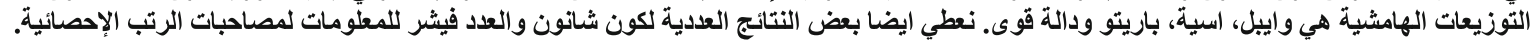

\section{Introduction}

Kamps [9] has introduced GOS's as a unified model of ordered random variables such as ordinary order statistics, sequential order statistics, progressive type-II censoring, record values and Pfeifers records. The joint density function of the GOS's $X(1, n, m, k), X(2, n, m, k), \ldots, X(n, n, m, k)$ is given by:

$$
\begin{aligned}
f^{X(1, n, m, k), \ldots, X(n, n, m, k)}\left(x_{1}, \ldots, x_{n}\right)= & k\left(\prod_{j=1}^{n-1} \gamma_{j}\right)\left(\prod_{i=1}^{n-1}\left(1-F\left(x_{i}\right)\right)^{m} f\left(x_{i}\right)\right) \\
& \times\left(1-F\left(x_{n}\right)\right)^{k-1} f\left(x_{n}\right), \\
& F^{-1}(0) \leq x_{1} \leq x_{2} \leq \cdots \leq x_{n} \leq F^{-1}(1),
\end{aligned}
$$

with parameters $n \in \mathbb{N}, k>0, m \in \mathbb{R}$, such that $\gamma_{r}=k+(n-r)(m+1)>0$, for all $1 \leq r \leq n$.

M. M. Mohie EL-Din · M. M. Amein

Department of Mathematics, Faculty of Science, Al-Azhar University, Cairo, Egypt

M. M. Amein

Department of Mathematics and Statistics, Faculty of Science, Taif University, Hawia, Kingdom of Saudi Arabia

N. S. A. Ali · M. S. Mohamed ( $\varangle)$

Department of Mathematics, Faculty of Education, Ain Shams University, Cairo, Egypt

E-mail: jin_tiger123@yahoo.com 
Morgenstern [12] has introduced FGM distributions; Gumbel [8] has studied FGM for exponential distribution. Farlie [5] has considered this family in the general form. Let $F_{X}(x)$ and $F_{Y}(y)$ be the distribution functions of the random variables $X$ and $Y$, respectively. Then the probability density function (pdf) of the bivariate FGM distributions is given by:

$$
f_{X, Y}(x, y)=f_{X}(x) f_{Y}(y)\left[1+\alpha\left(2 F_{X}(x)-1\right)\left(2 F_{Y}(y)-1\right)\right], \quad-1 \leq \alpha \leq 1 .
$$

Here, $f_{X}(x)$ and $f_{Y}(y)$ are the marginal pdf's of $X$ and $Y$, respectively. The parameter $\alpha$ is known as the dependence parameter of the random variables $X$ and $Y$. If $\alpha$ is zero, then $X$ and $Y$ are independent. For the FGM family with pdf given by (1.1), the density function of the concomitant of r-th GOS's $Y_{[r, n, m, k]}$, $1 \leq r \leq n$, is given by Beg and Ahsanullah [1], as follows:

$$
g_{[r, n, m, k]}(y)=f_{Y}(y)\left[1+\alpha C^{*}(r, n, m, k)\left(2 F_{Y}(y)-1\right)\right]
$$

where $C^{*}(r, n, m, k)=1-\frac{2 \prod_{j=1}^{r} \gamma_{j}}{\prod_{i=1}^{r}\left(\gamma_{i}+1\right)}$. Entropy is an index that is used to measure dispersion, volatility risk and uncertainty. This concept was formerly introduced by Shannon [13] in the information theory literature. The Shannon entropy of a random variable $X$ is a mathematical measure of information which measures the average reduction of uncertainty of $X$. Tahmasebi and Behboodian [15] have introduced the Shannon entropy for concomitants of GOS's of FGM family, the Shannon entropy for a continuous random variable $X$ with pdf $f_{X}(x)$ is defined as:

$$
H(X)=-E\left(\ln f_{X}(X)\right)=-\int_{-\infty}^{\infty} f_{X}(x) \ln f_{X}(x) \mathrm{d} x .
$$

Tahmasebi and Jafari [16] have introduced the Fisher information number for concomitants of GOS's of FGM family, the Fisher information number for a continuous random variable $X$ with pdf $f_{X}(x)$ is defined as:

$$
I(X)=\int_{-\infty}^{\infty}\left[\frac{\partial \ln f_{X}(x)}{\partial x}\right]^{2} f_{X}(x) \mathrm{d} x
$$

this is Fisher information number for location parameter, and also called shift-invariant Fisher information number. Furthermore, it has been used to develop a unifying theory physical law called the principle of "extreme physical information" (see Frieden [6,7]). Noting that, it is different than what was introduced by BuHamra and Ahsanullah [2].

Remark 1.1 In the computation of this paper, we use some important formulas as follow:

1. Let $T(t)=\int_{-\infty}^{\infty}[f(x)]^{t} \mathrm{~d} x$. Then $\left[-\frac{\partial T(t)}{\partial t}\right]_{t=1}=H(X), t \geq 1$.

2. Let $A(X)=\int_{-\infty}^{\infty} u(x) f(x) \ln f(x) \mathrm{d} x, U(t)=\int_{-\infty}^{\infty} u(x)[f(x)]^{t} \mathrm{~d} x$. Then $\left[\frac{\partial U(t)}{\partial t}\right]_{t=1}=A(X), t \geq 1$.

The rest of this article is organized as follows. In Sect. 2, we derive Shannon entropy for concomitants of GOS's of FGM family for some well-known distributions such as Weibull, Pareto and power function distributions. In Sect. 3, we develop Fisher information number for concomitants of GOS's of FGM family for some well-known distributions such as exponential, Pareto and power function distributions. In Sect. 4, we compute numerical values of our results for concomitants of order statistics.

\section{Shannon entropy for concomitants of GOS's from subfamilies of FGM family}

Tahmasebi and Behboodian [15] have introduced the Shannon entropy for concomitants of GOS's of FGM family by the following theorem:

Theorem 2.1 If $Y_{[r, n, m, k]}$ is the concomitant of $r$-th GOS's from (1.1), then, from (1.3), the Shannon entropy of $Y_{[r, n, m, k]}$ for $1 \leq r \leq n, \alpha \neq 0,-1 \leq \alpha \leq 1$ is given by:

$$
H\left(Y_{[r, n, m, k]}\right)=W(r, \alpha, n, m, k)+H(Y)\left(1-\alpha C^{*}(r, n, m, k)\right)-2 \alpha C^{*}(r, n, m, k) \phi_{f}(y),
$$

where

$$
\begin{aligned}
W(r, \alpha, n, m, k)= & \frac{1}{4 \alpha C^{*}(r, n, m, k)}\left[\left(1-\alpha C^{*}(r, n, m, k)\right)^{2} \ln \left(1-\alpha C^{*}(r, n, m, k)\right)\right. \\
& \left.-\left(1+\alpha C^{*}(r, n, m, k)\right)^{2} \ln \left(1+\alpha C^{*}(r, n, m, k)\right)\right]+\frac{1}{2}
\end{aligned}
$$




$$
\phi_{f}(y)=\int_{-\infty}^{\infty} F_{Y}(y) f_{Y}(y) \ln f_{Y}(y) \mathrm{d} y .
$$

In the following subsections, we will apply the last theorem to some subfamilies of FGM family such as Weibull, Pareto and power function distributions.

\subsection{Weibull distribution}

The pdf and cdf for Weibull distribution are given by, respectively:

$$
\begin{gathered}
f(y)=c y^{c-1} e^{-y^{c}}, \\
F(y)=1-e^{-y^{c}}, \quad 0 \leq y<\infty, c>0 .
\end{gathered}
$$

Theorem 2.2 If $Y_{[r, n, m, k]}$ is the concomitant of $r$-th GOS's for Weibull distribution from (1.1) and (2.5) then, from (2.1), the Shannon entropy of $Y_{[r, n, m, k]}$ for $1 \leq r \leq n, \alpha \neq 0,-1 \leq \alpha \leq 1$ is given by:

$$
\begin{aligned}
H\left(Y_{[r, n, m, k]}\right)= & W(r, \alpha, n, m, k)+\left(1+\alpha C^{*}(r, n, m, k)\right)\left[\frac{c-1}{c} v-\ln c+1\right] \\
& -\alpha C^{*}(r, n, m, k)\left[\frac{c-1}{c}(v+\ln 2)-\ln c+\frac{1}{2}\right],
\end{aligned}
$$

where $v=-\Gamma^{\prime}(1)$ is the Euler's constant and $W(r, \alpha, n, m, k)$ is defined by (2.2).

Proof From (2.1), (2.4) and (2.5), we have

$$
\begin{aligned}
H\left(Y_{[r, n, m, k]}\right)= & W(r, \alpha, n, m, k)-\left(1-\alpha C^{*}(r, n, m, k)\right) \int_{0}^{\infty} c y^{c-1} \exp \left(-y^{c}\right) \ln \left(c y^{c-1} \exp \left(-y^{c}\right)\right) \mathrm{d} y \\
& -2 \alpha C^{*}(r, n, m, k) \int_{0}^{\infty} c y^{c-1} \exp \left(-y^{c}\right)\left(1-\exp \left(-y^{c}\right)\right) \ln \left(c y^{c-1} \exp \left(-y^{c}\right)\right) \mathrm{d} y \\
= & W(r, \alpha, n, m, k)-\left(1+\alpha C^{*}(r, n, m, k)\right) \int_{0}^{\infty} c y^{c-1} \exp \left(-y^{c}\right) \ln \left(c y^{c-1} \exp \left(-y^{c}\right)\right) \mathrm{d} y \\
& +2 \alpha C^{*}(r, n, m, k) \int_{0}^{\infty} c y^{c-1} \exp \left(-2 y^{c}\right) \ln \left(c y^{c-1} \exp \left(-y^{c}\right)\right) \mathrm{d} y \\
= & W(r, \alpha, n, m, k)-\left(1+\alpha C^{*}(r, n, m, k)\right) I+2 \alpha C^{*}(r, n, m, k) I I .
\end{aligned}
$$

To find

$$
I=-H(Y)=\int_{0}^{\infty} c y^{c-1} \exp \left(-y^{c}\right) \ln \left(c y^{c-1} \exp \left(-y^{c}\right)\right) \mathrm{d} y,
$$

first, we want to obtain

$$
\begin{aligned}
T(t)= & \int_{0}^{\infty}[f(y)]^{t} \mathrm{~d} y=\int_{0}^{\infty} c^{t} y^{t(c-1)} \exp \left(-t y^{c}\right) \mathrm{d} y \\
= & c^{t-1} t^{-\left(\frac{t(c-1)+1}{c}\right)} \Gamma\left(\frac{t(c-1)+1}{c}\right) . \\
\Longrightarrow \frac{\partial T(t)}{\partial t}=T^{\prime}(t)= & Q c^{t-1}(\ln c) \Gamma\left(\frac{t(c-1)+1}{c}\right)+Q^{\prime} c^{t-1} \Gamma\left(\frac{t(c-1)+1}{c}\right) \\
& +Q c^{t-1}\left(\frac{c-1}{c}\right) \Gamma^{\prime}\left(\frac{t(c-1)+1}{c}\right),
\end{aligned}
$$


where $Q=t^{-\left(\frac{t(c-1)+1}{c}\right)}, Q^{\prime}=Q\left(-\left(\frac{t(c-1)+1}{t c}\right)-\left(\frac{c-1}{c}\right) \ln t\right)$.

$$
\Longrightarrow T^{\prime}(1)=I=\ln c-\frac{c-1}{c} v-1 \text {. }
$$

To find

$$
I I=\int_{0}^{\infty} c y^{c-1} \exp \left(-2 y^{c}\right) \ln \left(c y^{c-1} \exp \left(-y^{c}\right)\right) \mathrm{d} y
$$

first, we want to obtain

$$
\begin{aligned}
U(t)= & \int_{0}^{\infty} \exp \left(-y^{c}\right)[f(y)]^{t} \mathrm{~d} y=\int_{0}^{\infty} c^{t} y^{t(c-1)} \exp \left(-(t+1) y^{c}\right) \mathrm{d} y \\
= & c^{t-1}(t+1)^{-\left(\frac{t(c-1)+1}{c}\right)} \Gamma\left(\frac{t(c-1)+1}{c}\right) . \\
& \Longrightarrow U^{\prime}(1)=I I=\frac{\ln c}{2}-\frac{c-1}{c}\left(\frac{v+\ln 2}{2}\right)-\frac{1}{4}
\end{aligned}
$$

where $v=-\Gamma^{\prime}(1)=0.57722$ is the Euler's constant. By substituting (2.8) and (2.9) in (2.9), the result follows.

From Weibull distribution, we can get the Shannon entropy of other related distributions such as exponential and Rayleigh distributions by changing the parameters.

\subsection{Pareto distribution}

The pdf and cdf for Pareto distribution are given by, respectively:

$$
\begin{gathered}
f(y)=c y^{-(c+1)}, \\
F(y)=1-y^{-c}, \quad y \geq 1, c>0 .
\end{gathered}
$$

Theorem 2.3 If $Y_{[r, n, m, k]}$ is the concomitant of $r$-th GOS's for Pareto distribution from (1.1) and (2.11) then, from (2.1), the Shannon entropy of $Y_{[r, n, m, k]}$ for $1 \leq r \leq n, \alpha \neq 0,-1 \leq \alpha \leq 1$ is given by:

$$
\begin{aligned}
H\left(Y_{[r, n, m, k]}\right)= & W(r, \alpha, n, m, k)+\left(1+\alpha C^{*}(r, n, m, k)\right)\left[\ln \frac{1}{c}+\frac{1}{c}+1\right] \\
& -\alpha C^{*}(r, n, m, k)\left[\frac{c+1}{2 c}-\ln c\right]
\end{aligned}
$$

where $W(r, \alpha, n, m, k)$ is defined by (2.2).

Proof The proof is similar to the proof of Theorem 2.2.

\subsection{Power distribution function}

The pdf and cdf for Power distribution function are given by, respectively:

$$
\begin{gathered}
f(y)=c y^{c-1}, \\
F(y)=y^{c}, \quad 0 \leq y \leq 1, c>0 .
\end{gathered}
$$


Theorem 2.4 If $Y_{[r, n, m, k]}$ is the concomitant of $r$-th GOS's for Power distribution function from (1.1) and (2.14) then, from (2.1), the Shannon entropy of $Y_{[r, n, m, k]}$ for $1 \leq r \leq n, \alpha \neq 0,-1 \leq \alpha \leq 1$ is given by:

$$
\begin{aligned}
H\left(Y_{[r, n, m, k]}\right)= & W(r, \alpha, n, m, k)-\left(1-\alpha C^{*}(r, n, m, k)\right)\left[\ln c-\frac{c-1}{c}\right] \\
& -\alpha C^{*}(r, n, m, k)\left[\ln c-\frac{c-1}{2 c}\right],
\end{aligned}
$$

where $W(r, \alpha, n, m, k)$ is defined by (2.2).

Proof The proof is similar to the proof of Theorem 2.2.

\section{Fisher information number for concomitants of GOS's from subfamilies of FGM family}

Tahmasebi and Jafari [16] have introduced the Fisher information number for concomitants of GOS's of FGM family by the following theorem:

Theorem 3.1 If $Y_{[r, n, m, k]}$ is the concomitant of $r$-th GOS's from (1.1), then, from (1.4), the Fisher information number of $Y_{[r, n, m, k]}$ for $1 \leq r \leq n, \alpha \neq 0,-1 \leq \alpha \leq 1$ is given by:

$$
\begin{aligned}
I\left(Y_{[r, n, m, k]}\right)= & \int_{-\infty}^{\infty}\left[\frac{\partial \ln f_{Y}(y)}{\partial y}\right]^{2} f_{Y}(y)\left[1+\alpha C^{*}(r, n, m, k)\left(2 F_{Y}(y)-1\right)\right] \mathrm{d} y \\
& +4 \alpha C^{*}(r, n, m, k) \int_{-\infty}^{\infty} f_{Y}^{\prime}(y) f_{Y}(y) \mathrm{d} y \\
& +4\left[\alpha C^{*}(r, n, m, k)\right]^{2} \int_{-\infty}^{\infty} \frac{f_{Y}^{3}(y)}{1+\alpha C^{*}(r, n, m, k)\left(2 F_{Y}(y)-1\right)} \mathrm{d} y .
\end{aligned}
$$

In the following subsections, we will apply the last theorem for some subfamilies of FGM family such as exponential, Pareto and power function distributions.

\subsection{Exponential distribution}

The pdf and cdf for exponential distribution are given by, respectively:

$$
\begin{gathered}
f(y)=e^{-y}, \\
F(y)=1-e^{-y}, \quad 0 \leq y<\infty .
\end{gathered}
$$

Theorem 3.2 If $Y_{[r, n, m, k]}$ is the concomitant of $r$-th GOS's for exponential distribution function from (1.1) and (3.3) then, from (3.1), the Fisher information number of $Y_{[r, n, m, k]}$ for $1 \leq r \leq n, \alpha \neq 0,-1 \leq \alpha \leq 1$ is given by:

$$
\begin{aligned}
I\left(Y_{[r, n, m, k]}\right)= & \frac{\left(1+\alpha C^{*}(r, n, m, k)\right)^{2}}{2 \alpha C^{*}(r, n, m, k)}\left[\ln \left(-1-\alpha C^{*}(r, n, m, k)\right)-\ln \left(-1+\alpha C^{*}(r, n, m, k)\right)\right] \\
& -4 \alpha C^{*}(r, n, m, k) .
\end{aligned}
$$

Proof Let $d=\alpha C^{*}(r, n, m, k)$. From (3.1), (3.2) and (3.3), we have

$$
\begin{aligned}
I\left(Y_{[r, n, m, k]}\right) & =\int_{0}^{\infty}\left[e^{-y}\left[1+d\left(1-2 e^{-y}\right)\right]-4 d e^{-2 y}+4 d^{2} \frac{e^{-3 y}}{1+d\left(1-2 e^{-y}\right)}\right] \mathrm{d} y \\
& =\int_{0}^{\infty}\left[-8 d e^{-2 y}+\frac{(1+d)^{2}}{-2 d+(1+d) e^{y}}\right] \mathrm{d} y \\
& =-4 d+\frac{(1+d)^{2}}{2 d}[\ln (-1-d)-\ln (-1+d)] .
\end{aligned}
$$




\subsection{Pareto distribution}

Theorem 3.3 If $Y_{[r, n, m, k]}$ is the concomitant of $r$-th GOS's for Pareto distribution from (1.1) and (2.11) then, from (3.1), the Fisher information number of $Y_{[r, n, m, k]}$ for $1 \leq r \leq n, \alpha \neq 0,-1 \leq \alpha \leq 1$ is given by:

$$
\begin{aligned}
I\left(Y_{[r, n, m, k]}\right)= & c(c+1)^{2}\left[\frac{1}{c+2}+\alpha C^{*}(r, n, m, k)\left(\frac{1}{c+2}-\frac{1}{c+1}\right)\right]-2 \alpha C^{*}(r, n, m, k) c^{2} \\
& -\frac{\alpha C^{*}(r, n, m, k) c^{3}}{c+1}-\frac{\left(1+\alpha C^{*}(r, n, m, k)\right) c^{3}}{c+2} \\
& +\frac{\left(1+\alpha C^{*}(r, n, m, k)\right)^{2} c^{3}}{4 \alpha C^{*}(r, n, m, k)}\left[{ }_{2} F_{1}\left(\frac{2}{c}, 1 ; 1+\frac{2}{c} ; \frac{2 \alpha C^{*}(r, n, m, k)}{1+\alpha C^{*}(r, n, m, k)}\right)-1\right] .
\end{aligned}
$$

Proof Let $d=\alpha C^{*}(r, n, m, k)$. From (3.1), (2.10) and (2.11), we have

$$
\begin{aligned}
I\left(Y_{[r, n, m, k]}\right)= & c(c+1)^{2} \int_{1}^{\infty}\left[y^{-c-3}+d\left(y^{-c-3}-2 y^{-2 c-3}\right)\right] \mathrm{d} y-4 d c^{2}(c+1) \int_{1}^{\infty} y^{-2 c-3} \mathrm{~d} y \\
& +4 d^{2} c^{3} \int_{1}^{\infty} \frac{y^{-3 c-3}}{1+d\left(1-2 y^{-c}\right)} \mathrm{d} y \\
= & c(c+1)^{2}\left[\frac{1}{c+2}+d\left(\frac{1}{c+2}-\frac{1}{c+1}\right)\right]-2 d c^{2}+4 d^{2} c^{3} \int_{1}^{\infty} \frac{y^{-3 c-3}}{1+d\left(1-2 y^{-c}\right)} \mathrm{d} y \\
= & c(c+1)^{2}\left[\frac{1}{c+2}+d\left(\frac{1}{c+2}-\frac{1}{c+1}\right)\right]-2 d c^{2}+4 d^{2} c^{3} I I I .
\end{aligned}
$$

To find

$$
\begin{aligned}
I I I=\int_{1}^{\infty} \frac{y^{-3 c-3}}{1+d\left(1-2 y^{-c}\right)} \mathrm{d} y= & \int_{1}^{\infty}\left[-\frac{y^{-2 c-3}}{2 d}-\frac{1+d}{4 d^{2}} y^{-c-3}-\frac{(1+d)^{2}}{8 d^{3}} y^{-3}\right. \\
& \left.+\frac{(1+d)^{3}}{8 d^{3}} \frac{1}{y^{3}\left((1+d)-2 \mathrm{~d} y^{-c}\right)}\right] \mathrm{d} y \\
= & -\frac{1}{4 d(1+c)}-\frac{(1+d)}{4 d^{2}(2+c)}-\frac{(1+d)^{2}}{16 d^{3}}+\frac{(1+d)^{3}}{8 d^{3}} I V
\end{aligned}
$$

we want to obtain

$$
I V=\int_{1}^{\infty} \frac{1}{y^{3}\left((1+d)-2 \mathrm{~d} y^{-c}\right)} \mathrm{d} y=-\int_{1}^{\infty} y^{-3}\left(2 \mathrm{~d} y^{-c}-(1+d)\right)^{-1} \mathrm{~d} y,
$$

let $z=y^{-c} \Rightarrow \mathrm{d} y=\frac{-1}{c} z^{-\frac{1}{c}-1} \mathrm{~d} z$. Then

$$
\begin{aligned}
I V & =\frac{1}{c} \int_{1}^{\infty} z^{\frac{2}{c}-1}(2 \mathrm{~d} z-(1+d))^{-1} \mathrm{~d} z \\
& =\frac{1}{c} \int_{1}^{\infty} z^{\frac{2}{c}-1} \sum_{n=0}^{\infty}\left(\begin{array}{c}
-1 \\
n
\end{array}\right)(2 \mathrm{~d} z)^{n}(-(1+d))^{-1-n} \mathrm{~d} z \\
& =\frac{1}{c} \sum_{n=0}^{\infty}\left(\begin{array}{c}
-1 \\
n
\end{array}\right)\left(\frac{2 d}{1+d}\right)^{n}(-1)^{-1-n}(1+d)^{-1} \int_{1}^{\infty} z^{n+\frac{2}{c}-1} \mathrm{~d} z \\
& =\left[\frac{-1}{2(1+d)} \sum_{n=0}^{\infty}\left(\begin{array}{c}
-1 \\
n
\end{array}\right)(-1)^{-n} \frac{1}{n+\frac{2}{c}}\left(\frac{2}{c}\right)\left(\frac{2 d}{1+d} z\right)^{n} z^{\frac{2}{c}}\right]_{1}^{\infty} \\
& =\frac{1}{2(1+d)}{ }_{2} F_{1}\left(\frac{2}{c}, 1 ; 1+\frac{2}{c} ; \frac{2 d}{1+d}\right),
\end{aligned}
$$

where ${ }_{2} F_{1}(a, b ; c ; z)$ is the Gaussian or ordinary hypergeometric function. By substituting (3.8) in (3.7) and (3.7) in (3.6), the result follows. 


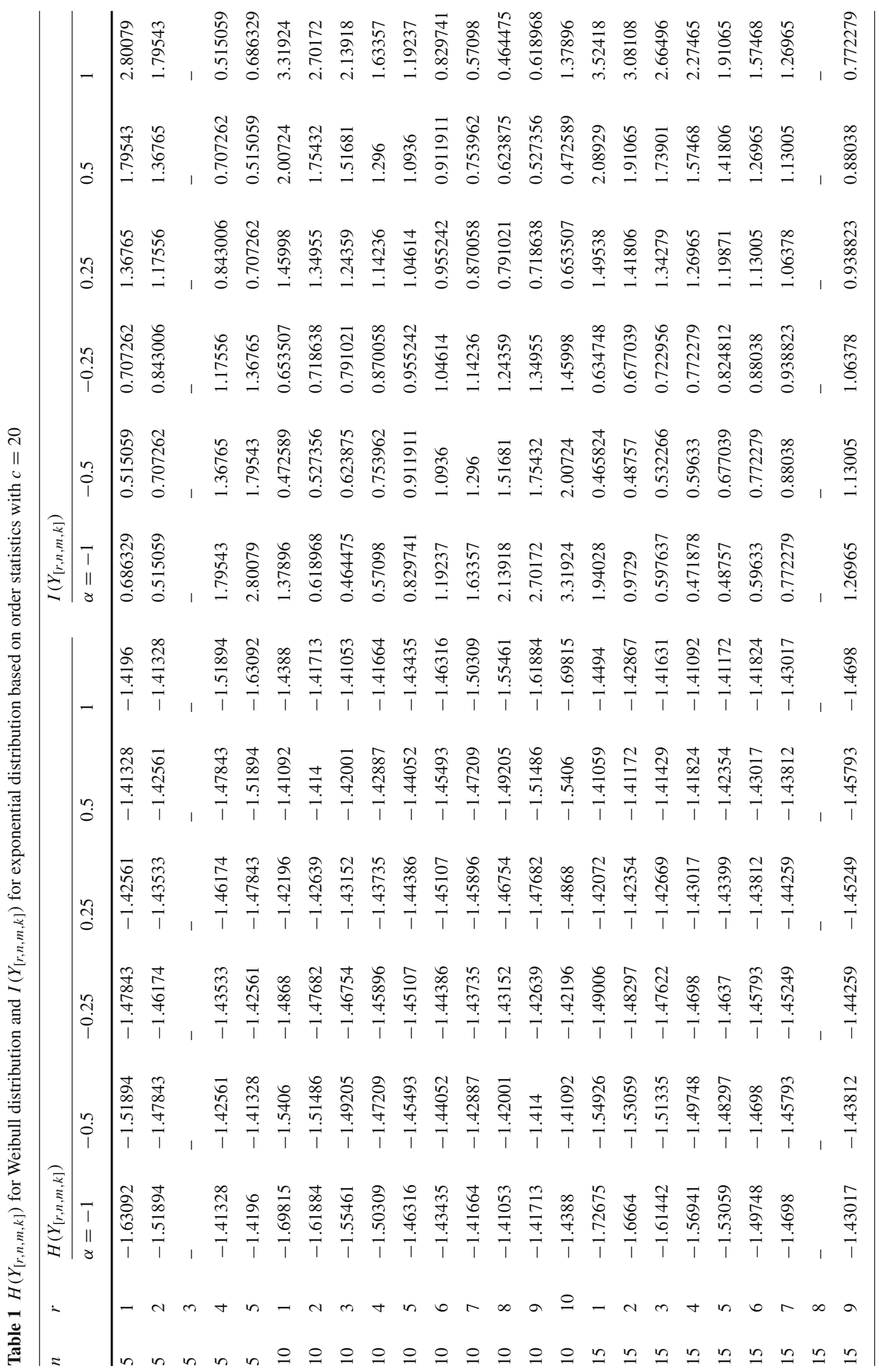

I 1 Springer 


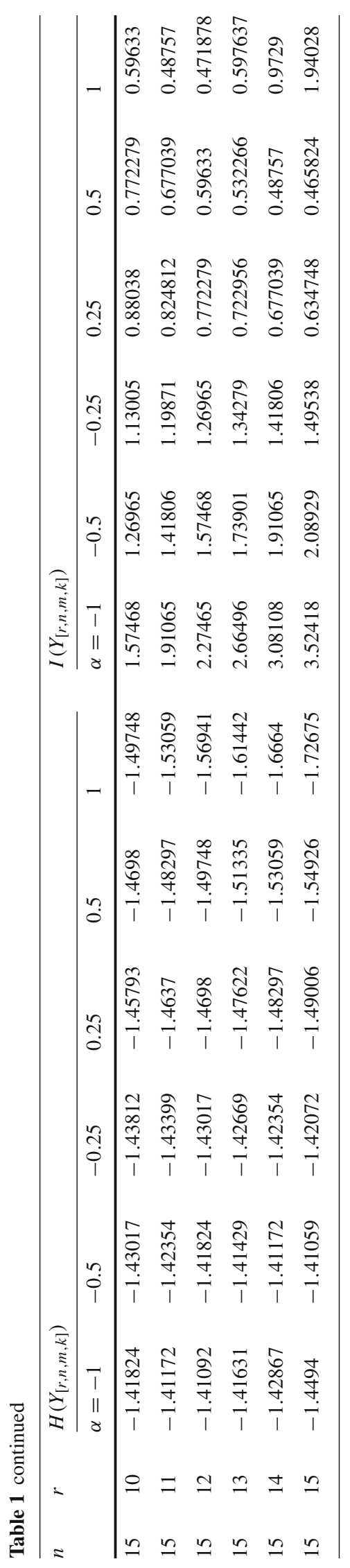

I- Springer 


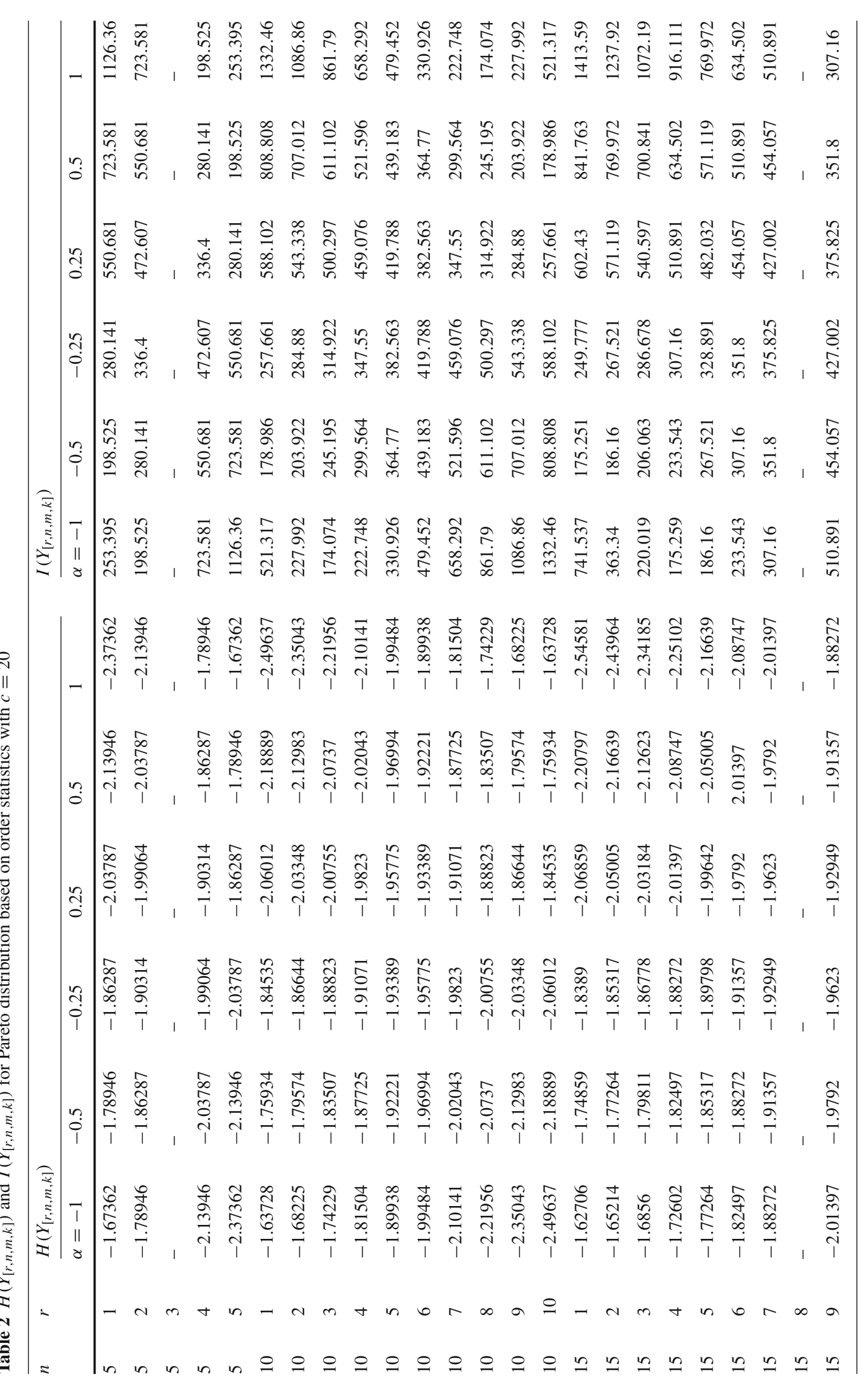




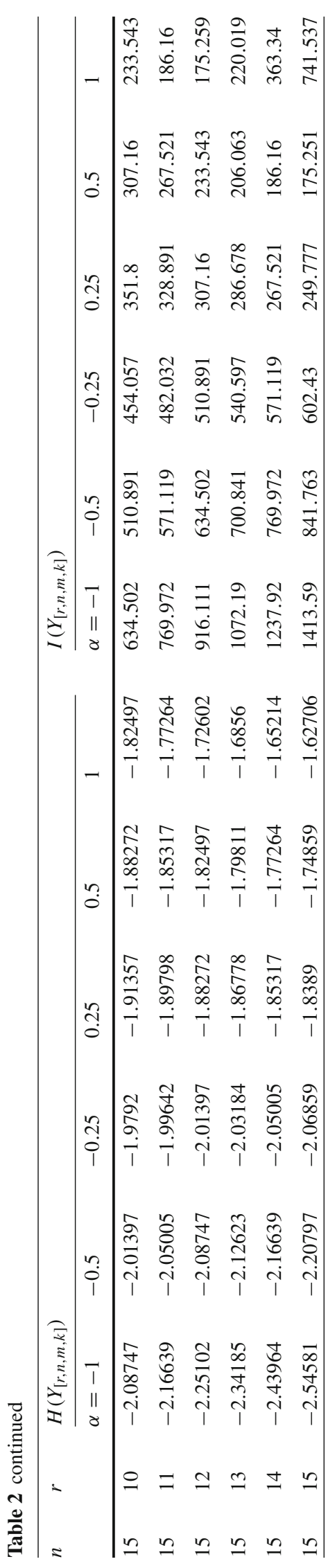

I Springer 


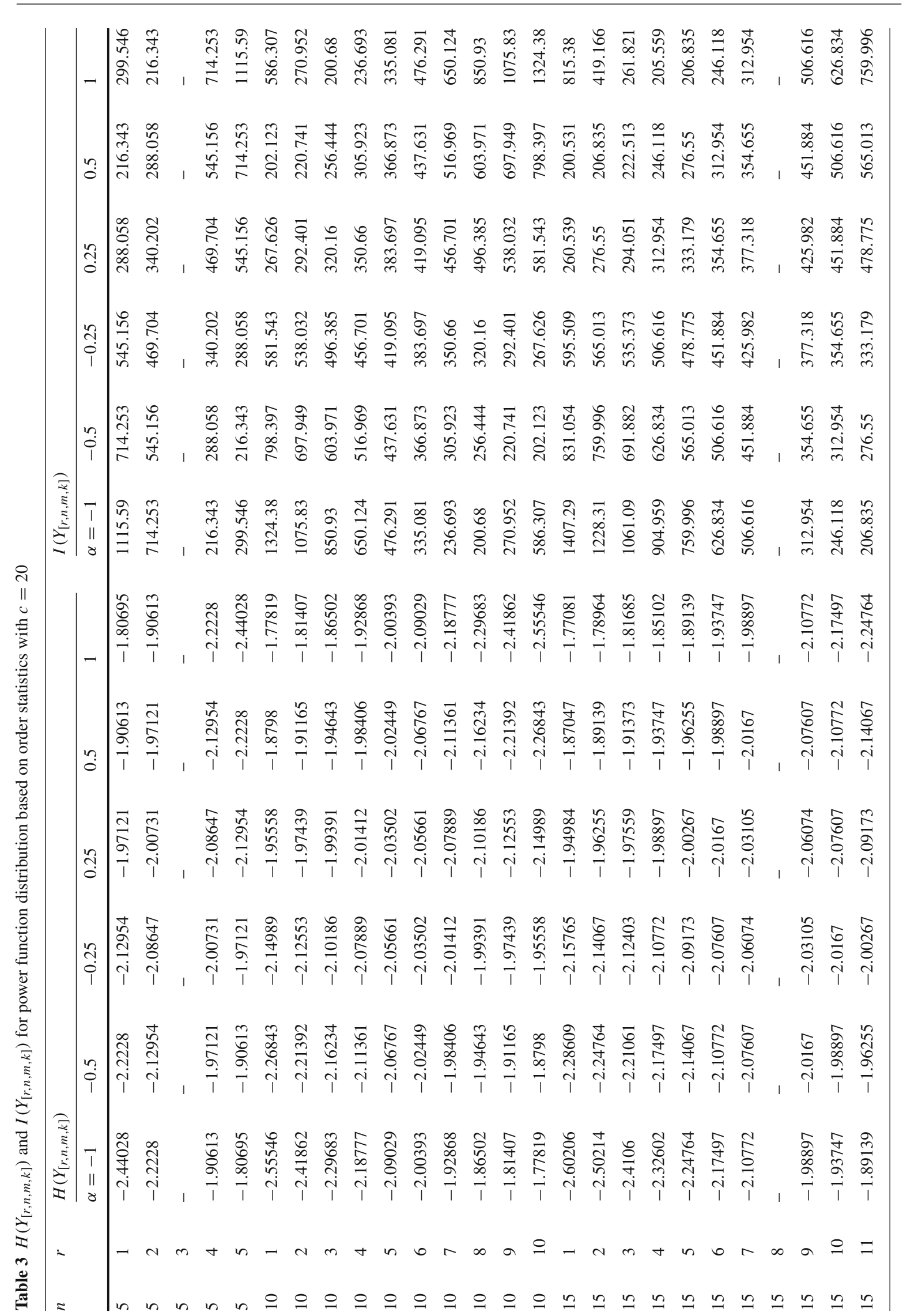




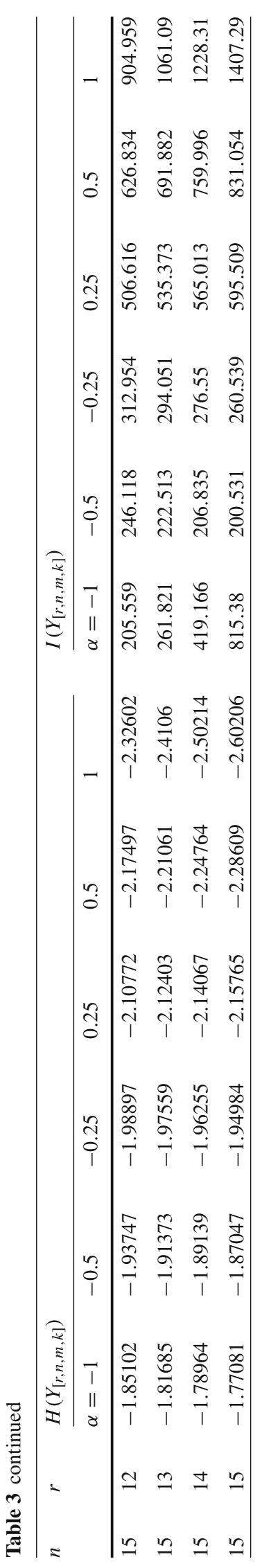

I Springer 


\subsection{Power distribution function}

Theorem 3.4 If $Y_{[r, n, m, k]}$ is the concomitant of $r$-th GOS's for Power distribution function from (1.1) and (2.14) then, from (3.1), the Fisher information number of $Y_{[r, n, m, k]}$ for $1 \leq r \leq n, \alpha \neq 0,-1 \leq \alpha \leq 1$ is given by:

$$
\begin{aligned}
I\left(Y_{[r, n, m, k]}\right)= & c(c-1)^{2}\left[\frac{1}{c-2}-\alpha C^{*}(r, n, m, k)\left(\frac{1}{(c-1)(c-2)}\right)\right]+2 \alpha C^{*}(r, n, m, k) c^{2} \\
& +\frac{c^{3}}{2 \alpha C^{*}(r, n, m, k)}\left[\frac{-\left(-1+\alpha C^{*}(r, n, m, k)\right)^{2}}{2}\right. \\
& +\frac{2 \alpha C^{*}(r, n, m, k)\left(-1+\alpha C^{*}(r, n, m, k)\right)}{c-2}+\frac{2\left(\alpha C^{*}(r, n, m, k)\right)^{2}}{c-1} \\
& \left.+\frac{\left(-1+\alpha C^{*}(r, n, m, k)\right)^{2}}{2}{ }_{2} F_{1}\left(\frac{-2}{c}, 1 ; 1-\frac{2}{c} ; \frac{-2 \alpha C^{*}(r, n, m, k)}{1-\alpha C^{*}(r, n, m, k)}\right)\right],
\end{aligned}
$$

$c \neq 1,2$.

Proof The proof is similar to the proof of Theorem 3.3.

\section{Numerical results}

Tables 1, 2 and 3 provide $H\left(Y_{[r, n, m, k]}\right)$ and $I\left(Y_{[r, n, m, k]}\right)$ values of the concomitants of order statistics $\left(\gamma_{i}=\right.$ $n-i+1$ ) when the marginal distributions are Weibull, exponential, Pareto and power function for $1 \leq r \leq n$, $n=5,10,15$ and $\alpha=-1,-0.5,-0.25,0.25,0.5,1$. We can find some properties from the numerical results as follow:

1. For $n$ is odd, $r=\frac{n+1}{2}$ we find that $H\left(Y_{[r, n, m, k]}\right)$ and $I\left(Y_{[r, n, m, k]}\right)$ are indeterminate.

2. Let $H\left(Y_{[r, n, m, k]}\right)=H_{\alpha}\left(Y_{[r]}\right)$ and $I\left(Y_{[r, n, m, k]}\right)=I_{\alpha}\left(Y_{[r]}\right)$. Then we have $H_{\alpha}\left(Y_{[r]}\right)=H_{-\alpha}\left(Y_{[n-r+1]}\right)$ and $I_{\alpha}\left(Y_{[r]}\right)$ $=I_{-\alpha}\left(Y_{[n-r+1]}\right), 1 \leq r \leq n$.

3. For fixed $n, r$, at $c>n, r<\frac{n+1}{2}\left(r>\frac{n+1}{2}\right)$ then we have, for Pareto distribution $H\left(Y_{[r, n, m, k]}\right)$ is decreasing (increasing) in $\alpha$, for power function distribution $H\left(Y_{[r, n, m, k]}\right)$ is increasing (decreasing) in $\alpha$.

4. For fixed $n, \alpha$, at $c>n, r \neq n,-1 \leq \alpha<0(0<\alpha \leq 1)$ then we have, for Weibull distribution $H\left(Y_{[r, n, m, k]}\right)$ is increasing (decreasing) in $r$, for Pareto distribution $H\left(Y_{[r, n, m, k]}\right)$ is decreasing (increasing) in $r$, for power function distribution $H\left(Y_{[r, n, m, k]}\right)$ is increasing (decreasing) in $r$.

5. For fixed $n, \alpha$, at $c>n,-0.5 \leq \alpha<0(0<\alpha \leq 0.5)$ then we have, for Weibull distribution $I\left(Y_{[r, n, m, k]}\right)$ is increasing (decreasing) in $r$, for Pareto distribution $I\left(Y_{[r, n, m, k]}\right)$ is increasing (decreasing) in $r$, for power function distribution $I\left(Y_{[r, n, m, k]}\right)$ is decreasing (increasing) in $r$.

6. For fixed $r, \alpha$, at $c>n,-1 \leq \alpha<0(0<\alpha \leq 1)$ then we have, for Weibull distribution $H\left(Y_{[r, n, m, k]}\right)$ is decreasing (increasing) in $n$, for Pareto distribution $H\left(Y_{[r, n, m, k]}\right)$ is increasing (decreasing) in $n$, for power function distribution $H\left(Y_{[r, n, m, k]}\right)$ is decreasing (increasing) in $n$.

7. For fixed $r, \alpha$, at $c>n,-0.5 \leq \alpha<0(0<\alpha \leq 0.5)$ then we have, for Weibull distribution $I\left(Y_{[r, n, m, k]}\right)$ is decreasing (increasing) in $n$, for Pareto distribution $I\left(Y_{[r, n, m, k]}\right)$ is decreasing (increasing) in $n$, for power function distribution $I\left(Y_{[r, n, m, k]}\right)$ is increasing (decreasing) in $n$.

8. For GOS's, we find that $-1 \leq C^{*}(r, n, m, k) \leq 1$ for all possible values of $r, n, m, k$.

\section{Conclusion}

We derived an analytical expression of Shannon entropy and Fisher information number from subfamilies of FGM family such as Weibull, exponential, Pareto and power distributions, based on concomitants of GOS's. Applications of these results are applied based on order statistics as a special case of GOS's. We find some important relations in entropy and Fisher information number at some values of the parameters. Conditions for decreasing (increasing) uncertainty and Fisher information number are obtained. We also observed the limit values of the constant $C^{*}(r, n, m, k)$ for GOS's. The proposed procedures may be considered for other models (such as dual generalized order statistics and case-II of generalized order statistics which are introduced by Burkschat et al. [3] and Kamps and Cramer [10], respectively), and for some other distributions. 
Open Access This article is distributed under the terms of the Creative Commons Attribution 4.0 International License (http:// creativecommons.org/licenses/by/4.0/), which permits unrestricted use, distribution, and reproduction in any medium, provided you give appropriate credit to the original author(s) and the source, provide a link to the Creative Commons license, and indicate if changes were made.

\section{References}

1. Beg, M.I.; Ahsanullah, M.: Concomitants of generalized order statistics from Farlie-Gumbel-Morgensterm distributions. Stat. Methodol. 5, 1-20 (2008)

2. BuHamra, S.S.; Ahsanullah, M.: Fisher information in concomitants of generalized order statistics in Farlie-GubmelMorgenstern distributions. J. Stat. Theory Appl. 4(4), 387-399 (2005)

3. Burkschat, M.; Kamps, U.; Cramer, E.: Dual generalized order statistics. Metron LXI(1), 13-26 (2003)

4. David, H.A., O'Connell, M.J.; Yang, S.S.: Distribution and expected value of the rank of a concomitant of an order statistic. Ann. Stat. 5, 216-223 (1977)

5. Farlie, D.J.G.: The performance of some correlation coefficients for a general bivariate distribution. Biometrika 47, 307-323 (1960)

6. Frieden, B.R.: Applications to optics and wave mechanics of the criterion of maximum Cramer-Rao bound. J. Mod. Opt. 35, 1297-1316 (1988)

7. Frieden, B.R.: Physics from Fisher Information-A Unification. Cambridge University Press, Cambridge (1998)

8. Gumbel, E.J.: Bivariate exponential distributions. J. Am. Stat. Assoc. 55, 698-707 (1960)

9. Kamps, U.: A concept of generalized order statistics. J. Stat. Plan. Inference 48, 1-23 (1995)

10. Kamps, U.; Cramer, E.: On distribution of generalized order statistics. Statistics 35, 269-280 (2001)

11. Mohie El-Din, M.M.; Amein, M.M.; Mohamed, M.S.: Concomitants of case-II of generalized order statistics from FarlieGumbel-Morgenstern distributions. J. Stat. Appl. Probab. 3, 345-353 (2015)

12. Morgentern, D.: Einfache beispiele zweidimensionaler verteilunngen. Mitteilungsblatt fur Mathemtische Statistik 8, 234235 (1956)

13. Shannon, C.E.: A mathematical theory of communication. Bell Syst. Tech. J. 27, 379-423 and 623-656 (1948)

14. Tahmasebi, S.; Behboodian, J.: Entropy for concomitants of order statistics in generalized Morgenstern (GM) sub-family and Pseudo-Weibull distribution. World Appl. Sci. J. 8, 789-791 (2010)

15. Tahmasebi, S.; Behboodian, J.: Shannon information for concomitants of generalized order statistics in Farlie-GumbelMorgenstern (FGM) family. Bull. Malays. Math. Sci. Soc. 35, 975-981 (2012)

16. Tahmasebi, S.; Jafari, A.A.: Fisher information number for concomitants of generalized order statistics in Morgenstern family. J. Inf. Math. Sci. 5, 15-20 (2013) 